\title{
Transporte y movilidad en la región de Chalco
}

\author{
Muriel Couturier* \\ Víctor Islas * *
}

Este trabajo pretende explorar las condiciones y la lógica de los traslados que realizan los habitantes en el municipio de Chalco y realizar una primera comparación con las propias características del transporte en toda el Área Metropolitana de la Ciudad de México (AMCM). Así, se analizan las formas y problemas que enfrentan los habitantes para desplazarse y se describen las principales características del transporte existente en esa región. Pero más que ello, se trata de explicar por qué los habitantes aceptan condiciones tan difíciles para el traslado cotidiano y por qué, a pesar de ello, se sigue incrementando la cantidad de nuevos asentamientos en la zona.

Una primera explicación radica en la combinación de dos factores: por una parte, el espíritu de sacrificio que los nuevos y jóvenes pobladores de Chalco muestran con el propósito de contar con vivienda propia y, por la otra, el relativo bajo costo de los terrenos. A lo anterior se agrega el hecho de que no todos los habitantes del municipio enfrentan verdaderas dificultades de traslado: hay habitantes que incluso disfrutan de muy amplias facilidades y hasta cierto exceso de transporte público, en función de contar con terrenos mejor ubicados ya sea por su precio o por su antigüedad. En todo caso, las deficiencias del transporte público y la deficiente y peligrosa vialidad interna y de conexión en las partes sur y poniente, hacen de Chalco un lugar que demuestra que la política de transporte urbano en México tiene graves rezagos e injusticias para la mayoría de la población.

En el plano teórico, parece comprobarse la propuesta de que las decisiones de localización de la vivienda están determinadas más por el acceso real al terreno (costo, facilidades, organización, etc.), que a una estrategia de minimización de costos y tiempos en el largo plazo, como ha sido más frecuentemente planteado.

\section{Introducción}

La percepción de los habitantes del municipio de Chalco sobre las cuestiones urbanas en general y los transportes en particular está determinada por la experiencia adquirida a partir de sus vivencias cotidianas, por la jerarquía de sus valores y de sus intereses. Es obvio que la percepción de sus habitantes no se expresa de la misma forma que la de la población del centro de la ciudad e inclusive de la del conjunto de habitantes de la Zona Metropolitana

\footnotetext{
* Université de Toulouse-Le Mirail.

* * Programa de Ciencia y Tecnología (Procientec), El Colegio de México.
} 
de la Ciudad de México. Sus percepciones están íntimamente ligadas a sus condiciones de vida fuertemente marcadas por las carencias en materia de transporte.

No es raro encontrar habitantes de la zona de Chalco que dediquen alrededor de cinco horas diarias para trasladarse a sus centros de trabajo y regresar a sus hogares. Sin embargo, a pesar de su lejanía con relación a las zonas industriales y de servicios del Área Metropolitana de la Ciudad de México, esta zona muestra un crecimiento realmente impresionante en los años recientes. En ese sentido, el presente artículo aborda el problema del traslado de pasajeros en la zona de Chalco y se cuestiona la forma un tanto anárquica en la que esta zona ha crecido y contribuido a deteriorar las condiciones de los viajes de sus habitantes. De hecho, en este trabajo se pretende avanzar en el estudio de la doble interrelación que se da entre el transporte y el desarrollo urbano: la existencia de transporte es una de las motivaciones para la expansión y diversificación de las actividades humanas en la urbe, pero también dicho crecimiento puede propiciar o demandar la expansión de las redes vial y de transporte. En este sentido, el de Chalco representa un caso de especial interés: atendiendo al razonamiento anterior (casi de libro de texto) se podría manejar la hipótesis de que son las favorables condiciones del transporte las que han hecho de esta zona una de las más atractivas para los nuevos asentamientos. Sin embargo, ése no parece ser el caso del municipio de Chalco. Así, se debe revisar esta hipótesis, y de no comprobarse, se debe buscar una explicación. En este trabajo se intenta dicho análisis aunque se reconoce que el tema del impacto que tiene el transporte en el desarrollo urbano está aún escaso de estudios serios y de metodologías prácticas. No obstante, es imposible negar el papel que han desarrollado los transportes en el crecimiento de la mancha urbana y en el tipo de usos del suelo que se van dando en la ciudad de México. En particular, es notable cómo han proliferado los asentamientos irregulares a partir de la extensión de nuevas rutas de taxis colectivos, Ruta 100 , autobuses suburbanos o el Aletro. También influye, y de forma determinante, el que estas zonas de la periferia del Distrito Federal, tengan un bajo costo de los predios. Así, una segunda hipótesis (la alternativa a la anterior], consiste en plantear que estos lugares son habitados aun a pesar de su difícil acceso (la red vial que conecta a la zona en estudio con el resto de la ciudad es deficiente y, en consecuencia, las rutas de transporte que sirven a estas colonias son pocas) merced al menor valor de los predios.

Cualquiera que sea la hipótesis más aceptable, es también claro que resultan necesarias diversas acciones que mejoren las ac- 
tuales condiciones del transporte de una de las zonas de menores ingresos por familia en toda el Área Metropolitana de la Ciudad de México. En ello, las características de la población deben ser un punto de partida para la definición de la política de transporte de la ciudad. Así, será importante identificar algunas propuestas específicas para mejorar dicha política de transporte.

El presente ensayo pretende analizar la situación del transporte en uno de los suburbios del Área Metropolitana de la Ciudad de México. Para ello, la primera parte describe sucintamente las principales características del sistema de transporte en la ciudad de México, así como una evaluación general del nivel de servicio que se está ofreciendo actualmente en cada modo de transporte. Dado ese contexto, la segunda parte revisa las principales características de lo que constituye de alguna manera el sistema de transporte de la zona de Chalco, tanto para su comunicación interna como para su vinculación con el resto del área metropolitana.

En la tercera parte del ensayo se presentarán algunos resultados preliminares relativos a las condiciones de desplazamiento de los habitantes del municipio de Chalco. Se utiliza como fuente de información una encuesta directa a una muestra de habitantes de la zona. ${ }^{1}$ Los resultados presentados aquí no pretenden ser representativos desde una perspectiva estadística. Sin embargo, los entrevistados fueron seleccionados considerando una dispersión espacial, aspecto fundamental cuando se trata de estudiar los desplazamientos. Así, los autores esperan poder aportar elementos de comprensión sobre las formas y condiciones de desplazamiento de los habitantes de la periferia urbana.

\section{El transporte en el Distrito Federal}

Para ubicarlos en su contexto y dimensionar mejor los problemas de transporte y vialidad en la región de Chalco, es necesario compararlos y ubicarlos dentro de la situación prevaleciente en el resto del área metropolitana o, más específicamente, en el Distrito Federal.

\footnotetext{
1 Esta encuesta fue realizada durante el periodo comprendido entre marzo y mayo de 1993 y forma parte de la tesis doctoral de Muriel Couturier, la cual se titula "Segregación urbana y uso del transporte colectivo en la periferia de la Zona Metropolitana de la Ciudad de México". Se realizaron 80 entrevistas a profundidad, las cuales fueron levantadas por Abraham Vilchis, becario asignado por el programa de Ciencia y Tecnología de El Colegio de México para esta tarea. En este documento, se presenta solamente una parte de los resultados concernientes al municipio de Chalco y las informaciones relativas a los desplazamientos al interior de este municipio.
} 
Actualmente, en la ciudad de México se tiene una situación problemática con relación al transporte y el desarrollo urbano con una diversidad de factores y consecuencias.

La ciudad de México se caracteriza por su gran extensión. Así, la superficie urbanizada ya rebasaba, en 1991, un total de $646.8 \mathrm{~km}^{2}$, aunque considerando el total del Área Metropolitana de la Ciudad de México (en adelante AMCM) la superficie de la mancha urbana continua se extiende en $1327.9 \mathrm{~km}^{2}$ (Departamento del Distrito Federal, 1993). Para 1990, el censo demográfico encontró que la población del AMCM casi llegaba a los 15 millones de habitantes, representando alrededor de $18 \%$ de la población total del país. La movilización de la población en la enorme superficie plantea graves problemas y un consumo impresionante de recursos humanos, energéticos y financieros. Al respecto cabe mencionar que al sector transporte, en los últimos años, se le ha asignado un promedio de 37\% del presupuesto total del Departamento del Distrito Federal (Islas, 1993).

Según el Anuario de vialidad y transporte de 1991, para ese año había un total de 2614695 vehículos en el Distrito Federal. Para atender la circulación de esa magnitud de vehículos la vialidad principal en el Distrito Federal consta de un sistema jerarquizado de vías entre las que destacan: ${ }^{2}$ algunas vías de acceso controlado (con un total de $141.3 \mathrm{~km}$ ), una retícula de arterias importantes conocidas como "ejes viales" con una longitud de $332.2 \mathrm{~km}$, algunas vías rápidas o principales (con una extensión de $972 \mathrm{~km}$ ), una gran cantidad de arterias secundarias $(8000 \mathrm{~km})$ y un conjunto de calles de menor capacidad.

Por otra parte, el sistema de transporte público se puede describir en forma muy sintética como muestra el cuadro 1 (Islas, 1993).

Existen varios modos de transporte de propiedad estatal: la empresa de autobuses conocida como Ruta 100, el Sistema de Transporte Colectivo-Metro (o sea, el clásico tren subterráneo con algunos tramos a nivel y elevados), y la empresa Servicio de Transportes Eléctricos.

La longitud total de kilómetros de rutas de que dispone Ruta 100, en ambos sentidos, rebasa la cifra de 7550 al finalizar el año de 1993. Esta red se extiende a lo largo de 216 rutas y se cuenta con unos 2480 autobuses para la operación (ya descontando los que están en reparación mayor o reconstrucción).

2 Según datos obtenidos de: DDF, El programa de mediano plazo 1985-1988 y Anuario de transporte y vialidad, 1991; Ing. Enrique Salcedo M., Transporte y vialidad en la ciudad de México. Experiencia de un sexenio, 1982-1988. 
CUADRO 1

Características del sistema de transporte urbano de pasajeros de la ciudad de México

\begin{tabular}{lcrrrr}
\hline & $\begin{array}{c}\text { Autobuses } \\
\text { Ruta-100 }\end{array}$ & $\begin{array}{c}\text { STC } \\
\text { Metro }\end{array}$ & $\begin{array}{c}\text { Trole- } \\
\text { buses }\end{array}$ & $\begin{array}{c}\text { Tren } \\
\text { ligero }\end{array}$ & $\begin{array}{c}\text { Taxis } \\
\text { colect. }\end{array}$ \\
\hline Rutas o líneas & 216 & 9 & 19 & 1 & 1200 \\
Longitud $(\mathrm{km})$ & 7556 & 158 & 440.2 & 25 & N.D. \\
Vehículos oper. & 2480 & 1647 & 269 & 54 & 44824 \\
\hline
\end{tabular}

Fuente: Quinto Informe de Gobierno 1993, México, Presidencia de la República; y "Diagnóstico preliminar del transporte en la ciudad de México", informe preparado por Víctor Islas para el Proyecto Transporte Urbano y Contaminación en la Ciudad de México, El Colegio de México, septiembre de 1993.

Por su parte, el Metro de la ciudad de México tiene un total de nueve líneas con una longitud de 158 kilómetros, 147 estaciones y 1647 carros en operación.

Finalmente, los otros transportes eléctricos (trolebuses y tren ligero) de la ciudad de México registran una presencia muy reducida: apenas alcanzaban para 1993 un total de 19 líneas de trolebuses con 440 kilómetros de rutas y 269 vehículos, y una línea de tren ligero con 25 kilómetros de extensión y 6 trenes en operación.

El transporte público prestado por particulares recae principalmente en los taxis colectivos que acumulaban para 1993, según las cifras oficiales, un total de 1200 ramales y casi 45000 vehículos. Sin embargo, algunos estudios anteriores ya indicaban un estimado de cuando menos 15000 kilómetros de ramales, y una flota de más de 100 mil vehículos.

La atención de la demanda, esto es, la distribución de viajes en los transportes públicos y privados, era para 1991 como se . muestra en el cuadro 2 , en el cual se observan las siguientes características:

a) Predomina el uso del transporte público frente al privado.

b) Los automóviles representan alrededor de $90 \%$ de los vehículos y apenas movilizan $13.6 \%$ de los viajes (aunque existen estimaciones que indican una menor participación).

c) Comparando estas cifras con las correspondientes a la década anterior, se concluye que los autobuses han disminuido drásticamente su participación mientras que los taxis colectivos y el STC-Metro la incrementan significativamente.

d) El único modo de transporte no motorizado, la bicicleta, tiene una participación mínima (en las estimaciones de $1991 \mathrm{ni}$ 
CUADRO 2

Distribución modal de los viajes-persona-día en el Área Metropolitana de la ciudad de México, 1991

\begin{tabular}{|c|c|c|}
\hline Modo de transporte & $\begin{array}{c}V P D \\
\text { (millones) }\end{array}$ & Porcentaje \\
\hline Sistema de transporte colectivo-Metro & 4.466 & 11.0 \\
\hline Autobuses urbanos (Ruta 100) & 3.700 & 9.1 \\
\hline Taxis colectivos (Distrito Federal) & 14.879 & 36.5 \\
\hline Taxis libres y de sitio & 2.296 & 5.6 \\
\hline Trolebús & 0.352 & 0.9 \\
\hline Tren ligero & 0.013 & - \\
\hline Subtotal serv. público (D.F.) & 25.706 & 63.1 \\
\hline Automóviles particulares & 3.800 & 9.3 \\
\hline Subtotal Distrito Federal & 29.506 & 72.4 \\
\hline Taxis colectivos (Estado de México) & 6.212 & 15.2 \\
\hline Taxis libres y de sitio (“) & 0.135 & 0.3 \\
\hline Autobuses suburbanos (“) & 3.150 & 7.7 \\
\hline Subtotal serv. público (“) & 9.497 & 23.3 \\
\hline Automóviles particulares (“) & 1.750 & 4.3 \\
\hline Subtotal Estado de México & 11.247 & 27.6 \\
\hline Total AMCM & 40.753 & 100.0 \\
\hline
\end{tabular}

Fuente: Anuario de Transporte y Vialidad, 1991. Coordinación General de Transporte, DDF, 1993.

siquiera fue considerado), mientras que en los modos no contaminantes (STC-Metro, trolebús, bicicleta y tranvía) no se realizan ni la tercera parte de los viajes.

e) Existe una diversidad de modos de transporte que se traduce en una enorme complejidad que elimina la posibilidad de soluciones generales o simplistas. Así, aunque no puede hablarse de un predominio absoluto de cierto modo de transporte, los taxis colectivos satisfacen ya a poco más de la mitad de los traslados motorizados. Además, tampoco hay predominio de una modalidad de propiedad (aunque sí de un uso preferentemente público del transporte). La conclusión de esto es la necesidad de desarrollar una política de transporte que reconozca y aproveche la diversidad de tecnologías y formas de organización que existen en el transporte de la ciudad.

En realidad, la distribución modal es un resultado directo de la política de transporte desarrollada en la ciudad de México: inversión en infraestructura, tarifas y subsidios, instrumentos de regulación, etc. El análisis detallado de estos instrumentos de política cae 
fuera del presente trabajo. Sin embargo, uno de los resultados de dicha política es la opinión de los usuarios, la cual se presenta en el cuadro 3. En él se hace una síntesis comparativa de las siguientes características en cada modo de transporte: tipo de propiedad, viajes por persona al día, tarifa, tiempo de espera, tiempo de acceso, seguridad, confort, facilidad de ascenso, trato al usuario, transbordos diarios por persona y opinión en general.

Las variables del cuadro 3 fueron obtenidas por medio de una encuesta de opinión realizada al azar entre 900 usuarios del transporte público, entre los meses de junio a agosto de 1993. Esta encuesta no proporciona información estadísticamente representativa, pero puede servir para tener una primera idea del nivel de servicio que perciben los usuarios.

El tiempo de espera se refiere a la cantidad de minutos que tiene que aguardar el usuario antes de poder ascender al vehículo, en la hora de máxima demanda. Es, evidentemente, un reflejo tanto de la frecuencia y tipo de despacho, como de la capacidad de los vehículos y del tamaño de la flota disponible en cada ruta o línea. Como señala el cuadro 3 , el modo de transporte que más hace esperar a sus usuarios son los autobuses: el doble de tiempo de los taxis colectivos, alrededor del triple de los trolebuses y cinco veces el del Metro. Esto se explica, principalmente, por la cantidad de usuarios que no pueden abordar los vehículos que ya pasan saturados. Incluso en el caso del Metro, aunque su frecuencia de paso es de poco más de un minuto, algunos de sus usuarios deben dejar pasar algunos trenes antes de poder abordar.

Por otra parte, la variable del tiempo de acceso a cada modo de transporte intenta medir el grado de cobertura que tienen sus redes. Así, se les preguntó a los usuarios la cantidad de minutos que tenían que emplear en el recorrido a pie para abordar el vehículo. En este sentido, el medio de transporte que tiene el menor tiempo de acceso es el Metro. Ello se explica porque la mayoría de sus usuarios llega a las estaciones en otro medio de transporte. Por el contrario, en muchos de los viajes por taxi colectivo, autobús o trolebús, estos modos son el inicio de dicho viaje. En particular, el bajo valor obtenido en los tiempos de acceso a los taxis colectivos es un reflejo de su mayor cobertura, pues penetran hasta las colonias más alejadas e inaccesibles y tienen una gran diversificación en sus ramales.

En las variables denominadas seguridad, confort, facilidad de ascenso y trato al usuario, se les solicitó a los usuarios que dieran su orden de preferencia: 1 al más seguro, 2 al siguiente, etc. Así, la cifra que aparece en la tabla indica el porcentaje con el que el modo de transporte en cuestión apareció en primer o segundo lugar y, por tal 
razón, no puede sumar $100 \%$ (de hecho, tampoco suma necesariamente $200 \%$, dado que la encuesta incluyó al transporte privado). De acuerdo con esta manera de evaluar a los modos de transporte, el modo de transporte más seguro es el Metro. ${ }^{3}$ Por su parte, sólo alrededor de $15 \%$ de los entrevistados considera seguros a los autobuses y taxis colectivos. En este último caso, el resultado no es sorprendente si se reconoce que los taxis colectivos desarrollan un tipo de conducción muy agresivo, dada la enorme competencia que tienen entre ellos, normalmente, para "ganar tiempo y pasaje". Además, un factor importante es la fragilidad de los vehículos que usan. Finalmente, aunque una cantidad muy reducida de usuarios considera seguros a los trolebuses, en realidad la mayoría de ellos no usan este modo de transporte y por ello pueden estar sesgadas sus respuestas.

Por el contrario, en el aspecto de "confort", los taxis colectivos resultan ser los más reconocidos, aunque sólo por poco más de la mitad de entrevistados. Este resultado se explica porque los taxis colectivos son el único modo de transporte público cuyos vehículos se detienen en casi cualquier lugar de la ruta, ahorrando recorridos a pie, escaleras, etc. Además, en muchos casos, el viaje se realiza sentado (aunque es claro que los taxis colectivos han perdido parte de esta ventaja con la introducción de minibuses cuyos asientos y altura interior del vehículo implican incomodidades). Detrás de los taxis colectivos, el Metro muestra un porcentaje de sólo $22 \%$ en la cantidad de usuarios que los considera confortables. Por el contrario, para el caso de los autobuses y trolebuses, casi sólo uno de cada diez usuarios los consideró así.

En lo referente a la facilidad de ascenso, destaca el gran porcentaje (88\%) de personas que encontró aceptable los casos de los taxis colectivos. Le sigue muy por detrás el Metro (uno de cada tres usuarios). En contraste, los usuarios tienen gran dificultad para ascender a los autobuses y trolebuses.

En el trato a los usuarios casi todos los modos de transporte público dejan qué desear. Los autobuses y el Metro tienen un nivel de aceptabilidad muy bajo para la mayoría de los usuarios: sólo en 12 y $32 \%$ de los casos, respectivamente, ocupan el primer o segundo lugar en preferencias en este aspecto. El caso de los taxis colectivos (TC) trae consigo una paradoja: a pesar de movilizar a la mayoría de los usuarios del transporte público, sólo aparecieron con casi $20 \%$

${ }^{3}$ De hecho, sólo se tiene información oficial de un alcance de trenes, y aunque se sabe (extraoficialmente) de un número indeterminado de accidentes personales, la cifra, al parecer, es baja en relación con la cantidad de usuarios que maneja el Metro. 
CUADRO 3

Factores del nivel de servicio y otras características de los principales modos de transporte de la ciudad de México, 1993

\begin{tabular}{lcccc}
\hline Característica & Ruta 100 & STC-Metro & Taxis C. & Trolebús \\
\hline Propiedad & Estatal & Estatal & Privada & Estatal \\
Tarifa (pesos) & 400 & 400 & 550 & 400 \\
Tiempo de espera & $11.3 \mathrm{~min}$. & 2.4 & 5.7 & 4.2 \\
Tiempo de acceso & $5.9 \mathrm{~min}$. & 3.4 & 4.2 & 5.9 \\
Seguridad & $15.1 \%$ & 52.6 & 15.8 & 11.3 \\
Confort & $10.7 \%$ & 22.1 & 51.9 & 10.2 \\
Facilidad de asc. & $11.6 \%$ & 31.4 & 88.2 & 4.6 \\
Trato al usuario & $12.1 \%$ & 31.6 & 19.7 & 2.9 \\
Menos transbordos & $\mathbf{8 0 . 3} \%$ & 65.6 & 19.7 & 33.3 \\
Opinión positiva & & & & \\
en general & $32.7 \%$ & 68.3 & 33.7 & 31.8 \\
\hline
\end{tabular}

Fuente: datos de pasajeros según el Quinto Informe de Gobierno, Presidencia de la República, 1993; el resto de la información proviene del "Diagnóstico preliminar del transporte en la ciudad de México", septiembre de 1993, y de la "Encuesta a usuarios del transporte público de pasajeros", junio-agosto de 1993, Proyecto Transporte Urbano y Contaminación en la Ciudad de México, El Colegio de México.

de las preferencias de los usuarios según el trato que se recibe de parte del conductor. Éste es, probablemente, uno de los peores aspectos de los TC, y requiere de un mayor control por parte de las autoridades, independientemente de las medidas de entrenamiento a conductores (en educación vial, relaciones humanas, etc). En cambio, la baja proporción de usuarios $(2.9 \%)$ que cree recibir buen trato en los trolebuses se debe a la igualmente baja cantidad de usuarios que realmente tienen acceso a su servicio, aunque es posible que también haya actitudes no amables de los operadores.

También se les cuestionó a los usuarios si consideraban que el servicio prestado por cada modo de transporte era adecuado, en general. Como se ve en el cuadro 3, apenas una tercera parte de los usuarios considera conveniente, en lo global, el servicio de los autobuses urbanos. Una gran mayoría consideró adecuado el servicio del Metro. En contraste, los taxis colectivos y el trolebús tienen una aceptación de tres de cada diez usuarios.

Nuevamente, una paradoja que surge de analizar este último aspecto, consiste en que si bien los taxis colectivos tienen una mala opinión global entre los usuarios, son cada vez más usados. La respuesta radica en dos hechos. Primero, que la opinión personal puede ser adversa, pero la necesidad de transportarse es ma- 
yor a cualquier opinión. Así, cuando se carece de alternativas para trasladarse, se usa lo único que se tiene disponible. Segundo, no puede ocultarse que, en realidad, los taxis colectivos tienen varios atributos que los hacen atractivos para el usuario: facilidad de ascenso, mayor cercanía a los lugares de destino o transbordo, mayor velocidad, etc. Sin embargo, es muy probable que muchos de sus actuales usuarios optarían por el servicio de los autobuses o trolebuses, pero éstos han estado limitados para atender la demanda.

Por otra parte, un hecho que es preocupante consiste en que, en general, la opinión de los usuarios no es todo lo favorable hacia los transportes públicos (excepto el caso del Metro) que se desearía para desalentar el uso del transporte privado. Si bien lo anterior puede parecer subjetivo, es innegable que las velocidades de recorrido son bajas o muy bajas, los tiempos de espera (especialmente el caso de los autobuses) son demasiado altos y el traslado es realmente no confortable en las horas de máxima demanda. Este problema resulta por sí mismo importante pues implica una ineficiencia que se transmite al resto del sistema económico. Pero aún puede añadirse una complicación adicional: el servicio de transporte público no puede, en esas condiciones, ayudar a reducir la creciente motorización de la ciudad y su secuela contaminante.

Para finalizar esta breve semblanza de las características operativas del transporte público en la ciudad de México, el cuadro 4 muestra diversas estimaciones de los tiempos de recorrido que tienen que consumir los habitantes de la ciudad de México para realizar sus desplazamientos cotidianos. Éste es un atributo muchas veces más importante que el pago de la tarifa por el servicio de traslado. Aunque depende esencialmente de las características tecnológicas de los vehículos y la infraestructura, también es influida por la forma como esté organizada la empresa prestataria y el servicio en general. Así, las diferencias entre un modo de transporte y otro pueden llegar a ser muy significativas. Sin embargo, por el momento lo que resulta de interés resaltar es el dato de los tiempos de traslado. Para 1972, la encuesta referida anteriormente encontró que, en una muestra de 71246 viajes, el tiempo promedio de recorrido fue de 40 minutos y 50 segundos. De hecho, es preciso notar (véase el cuadro 4) que en la distribución de frecuencias de los tiempos de traslado existe una tendencia a dispersarse alrededor del valor más frecuente $(30.36 \%$ de los viajes cae en el rango de 30 a 59 minutos) pero existe un relativo sesgo hacia los viajes de más de dos horas de traslado, los cuales representaban $10.64 \%$. Sin duda estos viajes se refieren a los desplazamientos 
que se realizaban entre la ciudad y las zonas que estaban en proceso de conurbación o que ya tenían un alto grado de dependencia de ella. Así, en aquellos años (1972) un desplazamiento hacia, por ejemplo, Cuautitlán o Los Reyes implicaba, ya considerando los transbordos y los recorridos dentro de la ciudad, tiempos mayores de las dos horas.

\section{CUADRO 4}

Distribución de los tiempos totales de traslado, en el Área Metropolitana de la ciudad de México

\begin{tabular}{lccc}
\hline Duración del viaje & $\begin{array}{c}1972 \\
\text { Porcentaje }\end{array}$ & $\begin{array}{c}1989 \\
\text { Porcentaje }\end{array}$ & $\begin{array}{c}1993 \\
\text { Porcentaje }\end{array}$ \\
\hline $01-15$ minutos & 20.26 & 2.8 & 4.6 \\
$15-29$ minutos & 28.61 & 2.9 & 10.5 \\
$30-59$ minutos & 30.36 & 28.3 & 25.7 \\
$60-120$ minutos & 10.13 & 46.1 & 36.8 \\
Más de 120 minutos & 10.64 & 19.9 & 22.4 \\
Total & 100.0 & 100.0 & 100.0 \\
Tiempo promedio & 40 '50" & $92^{\prime} 42^{\prime \prime}$ & 94 '36" \\
\hline
\end{tabular}

Fuente: 1972, cálculos propios basados en el "Estudio de transporte colectivo, 1972", Departamento del Distrito Federal; 1989, "Encuesta a usuarios del transporte público de pasajeros", Procientec, El Colegio de México; 1993, "Encuesta a usuarios del transporte público de pasajeros", Procientec, El Colegio de México.

Un problema de los datos de 1972 consiste en haber considerado también los viajes realizados a pie. Estos, por su naturaleza, son viajes relativamente cortos y a lugares cercanos. En cambio, si el análisis se centra en los viajes realizados entre diferentes zonas de la ciudad, se tiene una mejor apreciación de la demanda de viajes atendida por medios motorizados. Así, los estudios realizados en 1989 y 1993 no incluyen los viajes realizados totalmente a pie. Ello explica por qué en el cuadro 4 se tiene una baja participación de los viajes menores a 30 minutos. Además, tales estudios han encontrado que los tiempos de recorrido se concentran alrededor de la hora y media de duración. En particular, el sondeo realizado en 1993 encontró que poco más de la cuarta parte de los viajes implican más de dos horas de recorrido. Estos datos no son sorprendentes para un usuario cotidiano del transporte en el Distrito Federal. Sin embargo, dan una idea de la gran cantidad de horas-hombre y horas-muịer (igual o más valiosas que las primeras) que se consumen en los traslados dentro de la gran ciudad. 


\section{La situación del transporte en Chalco}

Aunque los habitantes de Chalco han quedado ya incluidos dentro de los usuarios que usan el sistema de transporte de la ciudad de México (también ya descrito), las cifras mencionadas no son, necesariamente, representativas de las condiciones específicas que enfrentan los usuarios dada la política de transporte que se desarrolla en Chalco.

Como toda política, la de transporte registra unos objetivos (explícitos o no), usa ciertos instrumentos y obtiene ciertos resultados. La falta o imprecisión en documentos oficiales hace que sea difícil ubicar los objetivos que tienen las autoridades en relación con el transporte de Chalco. Sin embargo, ellos pueden ser deducidos a partir de los instrumentos y sus resultados. Así, esta sección del artículo intenta describir algunas de las características físicas, operativas y tarifarias del transporte, dado el contexto geográfico y social en que se desenvuelven los usuarios. En la sección tercera se analizará lo correspondiente a los resultados de la política de transporte: el nivel de servicio que se ofrece a los usuarios.

\section{Chalco: ubicación y situación geográfica}

Chalco es uno de los 121 municipios que forman al Estado de México, con la clave de división político-administrativa 009 y clave geoestadística 025 .

Este municipio, que localmente recibe el nombre de Chalco de Díaz Covarrubias, se encuentra asentado en un valle, y tiene como límites por el norte al Canal de La Compañía y al río Miraflores. Estos límites le separan del municipio de Ixtapaluca. Sus zonas oriente y sur están formadas por zonas agrícolas y ganaderas que colindan con el estado de Morelos. En la zona poniente se encuentra la zona ejidal del Distrito Federal (Xochimilco y Tláhuac). En su zona suroeste se localiza el cerro de Xico que es su única elevación ( $2340 \mathrm{msnm}$ ). Chalco está ubicado en la latitud $19^{\circ} 15^{\prime} 53^{\prime \prime}$, y longitud $98^{\circ} 53^{\prime} 5^{\prime \prime}$ y a una altura promedio de $2300 \mathrm{me}-$ tros sobre el nivel del mar.

Esta localidad cuenta, según el censo demográfico de 1990, con una población cercana a los 300000 habitantes. Sin embargo, su expansión es más bien reciente. Un trabajo de Alicia Lindón indica que Chalco

presenta tres áreas claramente diferenciadas desde el punto de vista de las formas de ocupación del espacio. Una de ellas es la cabecera 
municipal, esto es el antiguo pueblo de Chalco, donde se reúne la mayor parte de la infraestructura urbana, vial y de transporte. Otra es la zona rural, situada en las zonas adyacentes de la cabecera municipal, y extendida al este de la misma. En la zona rural se encuentran algunas pequeñas localidades. La tercera zona es el valle de Chalco, el asentamiento de reciente urbanización emplazado en el noroeste del municipio (Lindón, 1991).

En efecto, el proceso de crecimiento de Chalco no se produjo en torno a la cabecera municipal, conocida como "Chalco antiguo" (véase el plano 1). Los asentamientos que realmente hacen crecer a Chalco empiezan hace quince años y se dan más bien en la zona norte del municipio en las zonas adyacentes a la carretera de cuota (colonias Tlalpizahuac, Avándaro, Del Carmen, etc.), y en los alrededores de Xico (colonias Xico, Guadalupana, etc.). En cambio, los asentamientos más recientes se están dando en la zona intermedia entre las dos mencionadas anteriormente. Así, colonias como Covadonga, Culturas de México, Nueva San Isidro, San Miguel y Tres Marías no tienen más de cuatro años de estarse desarrollando.

Estos últimos asentamientos establecen hoy una continuidad física con la cabecera municipal de Chalco.

\section{Descripción física del transporte}

La descripción de las condiciones actuales de la vialidad y el transporte constituyen el punto de partida para el análisis de la movilidad en esta región. La información contenida en esta sección y la siguiente proviene de una investigación realizada directamente en el municipio de Chalco, en los meses de junio a julio de 1993.

Por su ubicación, este municipio tiene ciertas facilidades en cuanto a la infraestructura vial orientada a los viajes interurbanos. Así, se cuenta con las siguientes carreteras o autopistas pavimentadas:

México-Puebla

Huitzilango-Distrito Federal

Tláhuac-Chalco

Mixquic-Chalco, San Nicolás Tetelco

Chalco-Tlalmanalco, San Mateo

Chalco-San Gregorio Cuautzingo, San Martín Cuautlalpan Además, se tienen los siguientes caminos de terracería:

San Miguel Xico-Chalco

San Miguel Xico Viejo-Amalinalco

Cuautlalpan-Huixtoco 


\section{PLANO 1}

\section{Ubicación de las colonias en el municipio de Chalco}

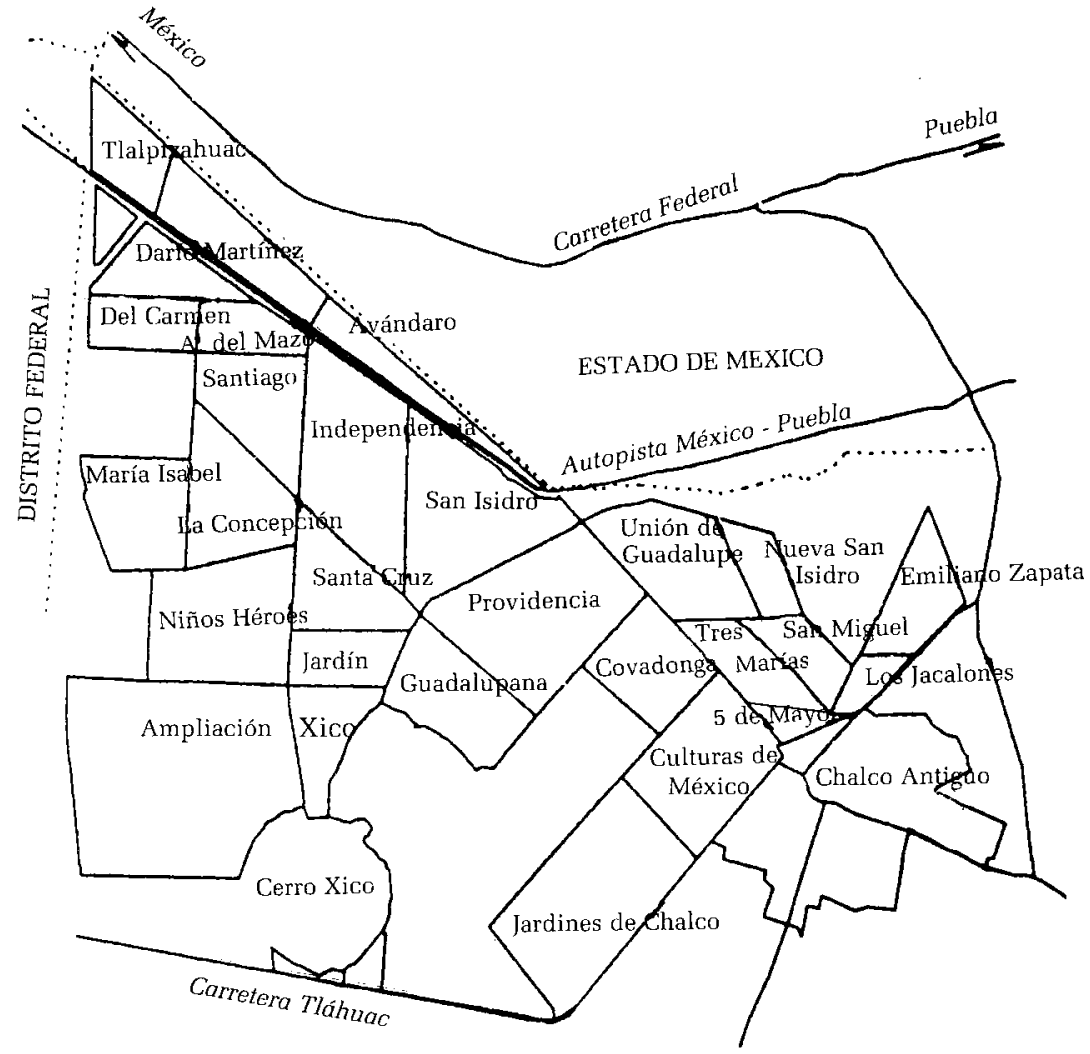

Fuente: Guía Roji, 1992, Coordinación de Servicios de Cómputo, El Colegio de México.

Una vía férrea atraviesa de noroeste a suroeste del municipio pasando por San Miguel Xico Viejo, San Lorenzo Chimalpa y San Pablo Atlazalpa.

Chalco es atravesado por autopistas, carreteras y avenidas, pero la mayor parte de calles que le sirven para su comunicación interna están sin pavimentar. En efecto, la pavimentación de calles y avenidas es una de las principales labores que actualmente 
ie está llevando a cabo en varias colonias mediante el programa le solidaridad. La zona que se observó con estos trabajos de pavinentación es la colonia de Xico donde se están colocando banjuetas y guarniciones.

Sobre los caminos de conexión con el Distrito Federal han surgido los siguientes comentarios.

1. Carretera federal México-Puebla (aunque realmente cruza jor el centro de Ixtapaluca).

Aunque resulta relativamente accesible, presenta una circulazión baja porque tiene graves problemas de mantenimiento preventivo y correctivo: los hoyos, "baches" y otras deficiencias son causa de una baja velocidad o el riesgo de accidentes.

2. Autopista México-Puebla.

Ésta es la mejor vía de comunicación existente en la zona y se le puede atribuir mucho del crecimiento de Chalco. Pasa cercana al límite entre Chalco e Ixtapaluca y se puede catalogar como una vía segura y rápida por ser autopista de cuota y presentar un mayor mantenimiento. Cabe mencionar que la gran mayoría de los vehículos que van a las nuevas zonas de Chalco no alcanzan a llegar a la caseta, pues se desvían antes. Así, disfrutan de una infraestructura a la que no contribuyen directamente a mantener en buenas condiciones.

3. Carretera de Chalco a San Pedro Tláhuac.

Ésta es una carretera muy importante para la población residente al suroeste de Chalco. Sin embargo, es de alto riesgo por cruzar una zona pantanosa. El problema radica en que, paradójicamente, esta carretera sólo está pavimentada en los tramos que no cruzan el lago. Así, el tramo que está sobre el agua se encuentra sin pavimentar y en época de lluvias este trayecto del camino es muy peligroso porque se producen deslaves de tierra. Además se enloda la vía principal y se dificulta o incluso interrumpe la circulación de vehículos. Esto ha generado varios accidentes, donde desafortunadamente se han perdido vidas humanas, debido principalmente a la volcadura de unidades al lago por las pésimas condiciones del camino. Cabe resaltar que este trayecto es recorrido por autobuses suburbanos que, en las malas condiciones de mantenimiento que tienen, no ofrecen ninguna seguridad a los usuarios del servicio.

4. Carretera Chalco-Mixquic.

Éste es un camino pequeño pero en relativo buen estado, en general. Sin embargo, por su orientación (hacia el sur) no atiende una mayor cantidad de viajes realmente interurbanos o hacia la ciudad.

Los caminos de comunicación de Chalco con otros estados sólo son dos: la autopista México-Puebla (que como se mencionó anteriormente está en buen estado y comunica con los estados de 
Puebla, Tlaxcala, Veracruz y Tabasco) y la carretera federal México-Cuautla, que es una vía de 2 carriles y está en muy mal estado, debido a la falta de presupuesto destinado al mantenimiento de las carreteras federales. Esta carretera comunica a este municipio con la ciudad de Cuautla, Morelos.

El transporte de este municipio está constituido por las modalidades de Microbuses, autobuses suburbanos "Chimecos", y por el servicio de Ruta 100. En menor cantidad se encuentra el servicio de taxis particulares. El servicio foráneo de autobuses es el que hace el traslado de gente del Distrito Federal a la zona centro de Chalco. En el cuadro 5 se indican las vías por las que transitan los diversos modos de transporte.

CUADRO 5

Principales arterias de uso por el transporte público

\begin{tabular}{lcccc}
\hline Vía de acceso & Ruta 100 & Suburbano & Microbús & Otro \\
\hline Carretera México-Puebla & & $\mathrm{X}$ & $\mathrm{X}$ & $\mathrm{X}$ \\
Autopista México-Puebla & $\mathrm{X}$ & $\mathrm{X}$ & $\mathrm{X}$ & $\mathrm{X}$ \\
Carretera México-Cuautla & $\mathrm{X}$ & & $\mathrm{X}$ & $\mathrm{X}$ \\
Carretera a San Pedro & $\mathrm{X}$ & & $\mathrm{X}$ & $\mathrm{X}$ \\
Carretera Chalco-Mixquic & $\mathrm{X}$ & & $\mathrm{X}$ & $\mathrm{X}$ \\
\hline
\end{tabular}

Fuente: observación directa.

El transporte que circula por la zona interior de Chalco transita principalmente por las siguientes avenidas: Cuauhtémoc, Vicente Guerrero, Vía de Prestigio, López Mateos, Isidro Fabela, Emiliano Zapata, Alfredo del Mazo y Nicolás Bravo. Cabe mencionar que la circulación presenta problemas en cuanto al mantenimiento de los vehículos puesto que los caminos en su mayoría están sin pavimentar, lo que provoca que en poco tiempo las unidades que transitan muy frecuentemente sufran averías y descomposturas lo que se agrava por tratarse de unidades viejas.

\section{Descripción operativa del transporte}

Como ya se mencionó en la anterior sección, la calidad del servicio de un modo de transporte se debe medir a partir de los atributos que de dicho modo de transporte requieren los usuarios. Tales atributos son muy diversos, pero los más frecuentes son: costo, velocidad, accesibilidad, cobertura, frecuencia, etc. (Islas, 1990). De una encuesta específica (que se describirá después) realizada 
ıntre los habitantes de Chalco, se encontró que los atributos prevalecientes para la selección del modo de transporte por los habiantes de esta región son la velocidad del servicio, la cercanía de los paraderos y el tiempo de espera.

Por ello, tales criterios deben guiar el análisis de la infraesructura y las rutas de transporte con que cuenta esta región para poder establecer cuáles son los mayores problemas. De la información recabada mediante entrevistas directas a los habitantes del municipio de Chalco, los principales problemas detectados son los siguientes:

En general, la demanda del transporte es muy alta, y el sistema de transporte no está integrado. Al contrario, existe mucha competencia entre los diversos modos de transporte, pero aun así no se satisface completamente la necesidad de la región.

Las calles no son continuas, en su gran mayoría, lo que provoca congestionamientos de tránsito muy frecuentes, sobre todo en las horas de máxima demanda, así como grandes demoras en los recorridos. Así, la condición deficiente de los caminos vuelve a tener un papel importante pues no permite circular a velocidades mayores a $50 \mathrm{~km} / \mathrm{hr}$. Ello tiene un aspecto favorable en cuanto a evitar accidentes, pero vuelve en realidad muy lento al servicio. Una mejor vialidad y medidas orientadas a una circulación segura (señalamiento, paradas, puentes peatonales, semáforos y otros dispositivos prácticamente ausentes en la región) resultan necesarias para mejorar los tiempos de traslado. Por otro lado, el problema de la saturación de los vehículos del transporte público provoca molestias a los usuarios. Ello incluso llega a ciertas agresiones verbales de parte de los operadores hacia los usuarios que se atreven a protestar por la política de llevar una excesiva cantidad de usuarios. Esto es particularmente cierto por parte de los operadores de los microbuses y camiones suburbanos y ocasiona un gran descontento porque además del alto costo del servicio, que repercute en la economía familiar, los tiempos de recorrido son muy largos porque se provocan demasiados retrasos por el ascenso y descenso de pasajeros. En este sentido una mejor alternativa de transporte en esta región es el servicio de Ruta 100, que llega a la zona en su categoría de expreso, y que resulta más económico y limpio. Sin embargo, este servicio presenta una grave falta de unidades para cubrir la demanda. El tiempo de despacho de las unidades disponibles es de 15 a 20 minutos y además sólo llegan a las colonias Zapata y Xico.

El trayecto de los viajes de entrada y salida al Distrito Federal, sobre todo en las horas pico, requiere de un recorrido de una a tres horas, lo que origina grandes horas-humanas perdidas por 
concepto de transporte. En efecto, debido a la gran cantidad de trabajadores dedicados a actividades secundarias, las horas de máxima demanda se presentan por las mañanas, a partir de las seis y hasta las nueve horas, lo que corresponde al horario de entrada de las industrias. El principal desplazamiento de la población es por motivo de trabajo: la mayoría de los usuarios viajan al exterior del municipio con destinos varios en las zonas industriales y de comercio del Distrito Federal. Igualmente, sus alternativas de regreso al hogar son mínimas, aunque se ha podido verificar que el tiempo requerido para transporte es mucho mayor si se desea ingresar al Distrito Federal que salir de él, con destino en la zona de Chalco. Este problema se debe a la insuficiente infraestructura vial que conecta a la región de Chalco con el Distrito Federal pues sólo se cuenta con dos caminos principales que son la autopista MéxicoPuebla y el camino hacia Tláhuac.

Los recorridos a pie también constituyen un grave problema. La ubicación de los paraderos tan alejados de las colonias de nueva creación, provoca que los usuarios tengan que caminar grandes distancias para hacer uso del servicio. También pierden tiempo esperando el paso de las unidades, ya que Ruta 100 no tiene suficientes unidades con itinerario de recorrido hacia esta zona, por lo que la frecuencia de paso puede variar de 5 a 40 minutos. Finalmente, el costo del transporte constituye un gasto muy fuerte para la economía familiar; en consecuencia es preferido el servicio de Ruta 100. Sin embargo, no parece ser un elemento muy considerado para la elección del modo de transporte. Lo que afecta es la falta de disponibilidad de parte de Ruta 100 que resulta insuficiente para satisfacer la demanda de este servicio en particular. Así, los habitantes de Chalco tienen que escoger modos de transporte más caros y por ello realizar un alto gasto en el servicio de transporte, lo que constituye una parte importante del gasto familiar. Ello es preocupante dado que el nivel económico de la región es más bien bajo. El cuadro 6 muestra las tarifas de un servicio específico para dar una idea de las mismas.

CUADRO 6

Costo del transporte en Chalco

\begin{tabular}{llll}
\hline Modo de transporte & Origen & \multicolumn{1}{c}{ Destino } & Precio \\
\hline Ruta 100 & Chalco & Metro San Lázaro & $40 \mathrm{cts}$ \\
Autobús suburbano & Chalco & Metro San Lázaro & $\mathrm{N} \$ 2$ \\
Microbús & Chalco & Metro San Lázaro & $\mathrm{N} \$ 2$ \\
Autobús foráneo & Chalco & Metro San Lázaro & $\mathrm{N} \$ 2.8$ \\
\hline
\end{tabular}

Fuente: datos recolectados en la zona (julio 1993). 
Hay un aspecto adicional que si bien no se traduce en desembolsos monetarios sí afecta los tiempos de desplazamiento y provoca molestias a los usuarios. Consiste en que las unidades de transporte a menudo son demasiado antiguas y están en mal estado, principalmente por la falta de mantenimiento, pero también por el polvo y el lodo que caracterizan el valle de Chalco en temporada de seca y de lluvia. ${ }^{4}$ Este mal estado se traduce no sólo en las inconveniencias o falta de confort, sino que es la causa de descomposturas, accidentes y otros males.

\section{Diferencias con el resto del transporte en el área metropolitana}

Aunque el objetivo del presente trabajo no es en sí una comparación de la política de transporte desarrollada en Chalco con la correspondiente a otras partes del Estado de México o del Distrito Federal, hay algunos elementos de análisis comparativo que resultan de mucha importancia. Antes de hacer tales observaciones, cabe notar que no es realmente correcto pensar en el transporte que opera en Chalco como una entidad separada o aislada del sistema de transporte de toda el área metropolitana ya que los modos de transporte de esta última complementan a los existentes en Chalco. Así, es el conjunto de modos de transporte lo que posibilita que parte de los habitantes de Chalco puedan llegar hasta sus lugares de destino en dicha área metropolitana y que tales habitantes se conviertan así en receptores de los efectos de la política de transporte que se desarrolla en el Distrito Federal, con las ventajas o desventajas que ello implica. No obstante, es en las características del transporte al interior de Chalco y en las dificultades para su conexión con el resto del área metropolitana que nos referiremos para efectos de comparación.

Los atributos del servicio que muestran evidentes diferencias son los siguientes: costo, tiempos de viaje, accesibilidad y alternativas modales.

En el costo, una comparación de los modos de transporte colectivo más usados en Chalco con relación al resto del AMCM revela que, en promedio, el usuario de Chalco enfrenta mayores costos: en efecto, considerando la cantidad de usuarios de cada modo de transporte y la tarifa que aplican (véase el cuadro 2), se obtiene un costo promedio mínimo por viaje de 0.61 nuevos pesos. Por su

${ }^{4}$ La zona de Chalco está ubicada en el antiguo lago de Tezcoco y resulta vulnerable a las inundaciones y a las nubes de polvo provocadas por la erosión. 
parte, aunque no se tienen estadísticas confiables de la cantidad de usuarios transportados en Chalco por cada modo de transporte, de la observación cotidiana se hace evidente que la mayoría de los usuarios selecciona a los taxis colectivos. Así, erogan alrededor de dos nuevos pesos sólo para llegar a su primer destino o a alguna estación del Metro.

En cuanto a los tiempos de recorrido, cabe recordar nuestra estimación de casi 95 minutos de viaje promedio para el AMCM (véase el cuadro 4). En cambio, aun con las ausencias de información, resulta claro que los usuarios de Chalco requieren entre 45 y 60 minutos tan sólo para llegar a la estación Zaragoza del Metro (el punto más frecuentemente usado para conectarse con el resto del sistema de transporte). Así, tal y como será ilustrado en la sección correspondiente a "tiempos y desplazamientos en el transporte colectivo" de este mismo trabajo, es probable que el tiempo de recorrido en promedio por cada viaje con origen en Chalco rebase la cifra de 120 minutos.

En la accesibilidad y las alternativas modales, nuevamente vuelven a estar los usuarios de Chalco en desventaja en relación con los niveles observados en el resto del AMCM. Aunque en estos atributos es difícil tener una medición incluso para el conjunto del área metropolitana, nuestras estimaciones indican que el usuario típico de los principales modos masivos del Distrito Federal requiere de un máximo de seis minutos de recorrido a pie para llegar al primer modo de transporte (véase el cuadro 2). Esto es resultado de la cobertura que en su conjunto proporcionan los taxis colectivos, el Metro, los autobuses y los trolebuses. Sin embargo, esta situación no es uniforme a lo largo del AMCM: mientras que el centro histórico de la ciudad de México concentra infraestructura y servicios de transporte, hay otras zonas con fuertes carencias. Ese es el caso de Chalco en donde es común encontrar zonas con una accesibilidad muy baja o nula: el recorrido a pie es la única forma de salir de la colonia aunque implique recorridos de hasta media hora.

Finalmente, y en buena medida como explicación de las diferencias observadas en los párrafos anteriores, es evidente que la cantidad de opciones modales en la zona (esto es, de tecnologías o formas de traslado) es mucho más reducida en Chalco que en el promedio del AMCM. Así, no se cuenta con ninguna línea de transporte masivo (Metro, tren ligero, etc.), ni con líneas de trolebuses. Por su parte, otros modos de transporte tienen una presencia mínima por diferentes razones: no hay facilidades para la bicicleta, Ruta 100 tiene una baja cantidad de rutas y vehículos (aunque sus servicios sean muy apreciados por los usuarios en la zona) y el 
automóvil no está al alcance de los ingresos de los habitantes de la zona ni cuenta con una vialidad razonable. Así, las únicas opciones reales son los taxis colectivos y, en menor medida, los autobuses suburbanos.

Las razones de la anterior situación son varias, y sólo las más importantes serán abordadas en el presente trabajo.

\section{Necesidades y actitudes de los usuarios del transporte colectivo en Chalco}

Los desplazamientos cotidianos no se reducen solamente a seleccionar un modo de transporte según el precio a pagar, sino también tienen una finalidad: acceder a los diferentes sectores de la ciudad a un cierto costo. Al gasto monetario hay que agregar el costo del tiempo empleado y el costo inherente a la incomodidad.

En las grandes ciudades de América Latina y más específicamente en las periferias de ellas, la necesidad de trasladarse se resuelve de un modo muy deficiente: con unidades en muy mal estado físico y de mantenimiento, excesiva saturación que llega a límites infrahumanos, irregularidad o bajísima frecuencia de paso, difícil acceso al servicio, etcétera.

Para tener una primera idea de las condiciones en que se realizan los viajes, se levantó una encuesta de características limitadas (es decir, sin pretensiones de representatividad estadística): se aplicó un total de 80 cuestionarios distribuidos en dos delegaciones del Distrito Federal (Azcapotzalco e Iztapalapa) y dos municipios del Estado de México (Chalco y Tlalnepantla). La selección del domicilio para aplicación de la encuesta se dejó al criterio del encuestador, sólo marcando como requisito que existiera una distancia de recorrido a pie de al menos 20 minutos entre domicilios para que las entrevistas quedaran lo más espaciadas posible dentro del municipio o delegación. Además, se procuró que al menos la mitad de las entrevistas fueran hechas con trabajadores y el resto se distribuyera entre amas de casa y estudiantes. Esto se hizo para tratar de analizar a los usuarios cautivos del transporte público. En el presente trabajo sólo se reporta lo referido al municipio de Chalco. Así, en la presente sección se presenta el análisis de la situación del transporte público, según las opiniones emitidas por los habitantes de Chalco a lo largo de las entrevistas. Dada la falta de representatividad estadística o la diversidad y complejidad de algunas respuestas (cabe aclarar que parte del cuestionario contenía preguntas abiertas y muy cualitativas), hemos reducido la elaboración de cuadros a sólo los realmente significativos. Creemos 
que, de las respuestas obtenidas, pueden plantearse preguntas y estudios más elaborados o más precisos.

El cuadro 7 señala la ocupación de las personas entrevistadas: catorce trabajadores, cuatro amas de casa y dos estudiantes con sus respectivos motivos de viaje (sólo se consideraron el trabajo, compras y estudios). El origen del viaje corresponde al lugar de vivienda, es decir, las diversas colonias de Chalco. Se puede observar que de los 20 casos, diez se dirigen hasta las delegaciones del Distrito Federal, seis van al interior de Chalco y cuatro tienen por destino otros municipios del Estado de México.

CUADRO 7

Datos básicos de los viajes detectados

\begin{tabular}{|c|c|c|c|}
\hline Ocupación & $\begin{array}{c}\text { Motivo de } \\
\text { viaje }\end{array}$ & $\begin{array}{l}\text { Origen: colonia } \\
\text { de Chalco }\end{array}$ & Destino \\
\hline 1 Empleado & Trabajo & Santa Cruz & Municipio Tlalmanalco \\
\hline 2 Obrero & Trabajo & Los Jacalones & Municipio La Paz \\
\hline 3 Vendedor & Trabajo & Emiliano Zapata & Delegación Cuauhtémoc \\
\hline 4 Ama de casa & Compras & San Sebastián & Chalco Antiguo \\
\hline 5 Chofer & Trabajo & Emiliano Zapata & $\begin{array}{l}\text { Delegación Gustavo A. } \\
\text { Madero }\end{array}$ \\
\hline 6 Ama de casa & Compras & $\begin{array}{l}\text { La Bomba } \\
\text { Chalco Antiguo }\end{array}$ & $\begin{array}{l}\text { Centro Chalco } \\
\text { Antiguo }\end{array}$ \\
\hline 7 Plomero & Trabajo & $\begin{array}{l}\text { La Conchita, } \\
\text { Chalco Antiguo }\end{array}$ & La Conchita \\
\hline 8 Ama de casa & Compras & Nueva San Isidro & Delegación Cuauhtémoc \\
\hline 9 Estudiante & Estudios & $\begin{array}{l}\text { Culturas de } \\
\text { México }\end{array}$ & Delegación Tlalpan \\
\hline 10 'Mil usos' & Trabajo & Covadonga & $\begin{array}{l}\text { Delegación Magdalena } \\
\text { Contreras }\end{array}$ \\
\hline 11 Albañil & Trabajo & Covadonga & Delegación Iztapalapa \\
\hline 12 Zapatero & Trabajo & $\begin{array}{l}\text { Culturas de } \\
\text { México }\end{array}$ & Chalco Antiguo \\
\hline 13 Machetero & Trabajo & Covadonga & $\begin{array}{l}\text { Delegación Miguel } \\
\text { Hidalgo }\end{array}$ \\
\hline 14 Albañil & Trabajo & Covadonga & Nueva San Isidro \\
\hline 15 Albañil & Trabajo & Providencia & Delegación Iztapalapa \\
\hline 16 Carretillero & Trabajo & Guadalupana & Delegación Iztapalapa \\
\hline 17 Ama de casa & Compras & San Miguel Xico & Xico Viejo \\
\hline 18 Soldador & Trabajo & Independencia & $\begin{array}{l}\text { Municipio Atizapán } \\
\text { de Zaragoza }\end{array}$ \\
\hline 19 Obrero & Trabajo & Santiago & Municipio Naucalpan \\
\hline 20 Estudiante & Estudios & Independencia & Delegación Cuauhtémoc \\
\hline
\end{tabular}

Fuente: datos recolectados en la zona (julio 1993). 
De los catorce trabajadores, siete laboran en el Distrito Federal, tres en Chalco y cuatro en otros municipios del Estado de México. Esta información es congruente con las estadísticas de una encuesta socioeconómica realizada en 1990 (Juárez y Martínez, 1990) en el valle de Chalco, que establece varios porcentajes relativos a la población activa: $60 \%$ trabaja en el Distrito Federal, más de $30 \%$ en el Estado de México, y menos de $10 \%$ en otros estados de la República. La distribución de la población según la actividad o el empleo es muy clara, ya que la mayoría son asalariados ya sea en la industria, en el comercio o esencialmente en los servicios.

La zona de Chalco ofrece pocos empleos y esto explica por qué una parte importante de la población se desplaza fuera de Chalco para ir a trabajar. De hecho, el transporte colectivo tiene importancia crucial en la vida cotidiana de los habitantes de Chalco.

\section{Percepción de los usuarios sobre el transporte colectivo}

Los principales problemas ligados al transporte colectivo pueden ser agrupados en tres tipos de respuestas.

El primero concierne al transporte colectivo en general: "malos servicios". El segundo tipo se refiere al comportamiento del chofer: "grosero, arbitrario en la fijación de las tarifas y de las paradas, y conducción peligrosa". El tercer tipo agrupa diferentes aspectos: "exceso de gente, cobertura deficiente, paradas lejanas al lugar de habitación, lentitud, mal estado del vehículo".

Los problemas ligados a la contaminación y a la comodidad (como por ejemplo el viaje de personas paradas) no fueron planteados por los habitantes de Chalco. La ausencia de esas preocupaciones subraya la gravedad de la situación del transporte colectivo en el municipio de Chalco: la cobertura insuficiente, el acceso difícil y las malas y aun peligrosas condiciones de traslado muestran que el transporte público actual no responde a las necesidades elementales de la población interrogada. Assí, la población reivindica un derecho elemental (el derecho a la movilidad) que no está satisfecho. Esto está confirmado por los requisitos que ellos consideran que tendría un buen transporte: eficiente, rápido, más unidades de transporte y en buen estado, y un transporte económico.

Las respuestas acerca de los diferentes medios de transporte usados revelan el hecho de que no es posible seleccionar el medio de transporte que les gustaría usar: se usa lo que hay. En efecto, los modos de transporte más criticados (incluso odiados), son los más usados. Se trata de los autobuses suburbanos y los taxis colectivos. Las personas entrevistadas caracterizan esos dos modos 
como los peores que cubren la zona: el suburbano, con unidades en mal estado y pésimo trato al usuario; las "combis" y microbuses, además de esas dos características, tienen un exceso de paradas.

El caso de la Ruta 100 es muy interesante, porque es un modo de transporte relativamente muy usado a pesar del reducido número de unidades y de una escasa cobertura en el espacio. Este modo de transporte es muy apreciado por la población de Chalco por su bajo costo (40 centavos, cualquiera que sea la distancia) y una relación chofer-usuario bastante cordial.

Una preocupación permanentemente expresada por los entrevistados se refiere al costo del transporte: casi todos coinciden en necesitar "transporte barato". La Ruta 100 es el único modo de transporte cuyo boleto es accesible para una población de bajos ingresos. Las tarifas de los otros medios, suburbano, microbuses y "combis" varía de uno a dos nuevos pesos (según la distancia recorrida), y el transporte "foráneo" (autobuses que pasan por la autopista México-Puebla) para ir al Distrito Federal alcanza hasta 2.5 nuevos pesos.

La diferencia de tarifas del transporte colectivo entre el Distrito Federal y el Estado de México es importante, tanto más para una población de bajos ingresos (entre cero y dos salarios mínimos) como la gran mayoría de los habitantes de Chalco (Juárez y Martínez, 1990).

Las personas encuestadas conocen esta diferencia de precio. Una cuarta parte la justifica por el mal estado de la vialidad en Chalco, y como un precio a pagar para entrar al Distrito Federal. Pero la mayoría estima que esta diferencia de tarifas es injusta porque: "los trayectos son los mismos", "la gente del Estado de México tiene menos ingresos y falta de servicios en general", "la mano de obra va a trabajar al Distrito Federal", "tenemos en gene$\mathrm{ral}$ condiciones de vida peores que los que viven en el Distrito $\mathrm{Fe}$ deral", "todos somos iguales, pertenecemos a la ciudad", etcétera.

La diferencia de pertenecer al Distrito Federal o al Estado de México tiene muy marcadas implicaciones para los usuarios del transporte. Y aunque no son residentes del Distrito Federal, la mayoría de los habitantes de Chalco que trabajan o estudian allí demandan la misma accesibilidad económica y espacial al transporte colectivo.

\section{Tiempos y desplazamientos en el transporte colectivo}

Las personas entrevistadas declararon que el "tiempo" interviene constantemente -“tarda mucho”, "es más rápido”, etc. Para ellos, el problema básico del desplazamiento se convierte en tiempo. 
Un análisis de la actividad de desplazamiento origen-destino realizado por medio de la medición del tiempo permitió captar vacios tipos de información. Cabe resaltar que se encontraron respuestas relativamente contradictorias. En efecto, la observación de las diferentes secuencias del desplazamiento cotidiano de ida, es decir, salida del domicilio-llegada al lugar de destino, y la respuesta a la pregunta sobre el tiempo de desplazamiento correspondiente, indica una dificultad para contabilizar el tiempo de traslado por parte de las personas interrogadas. Esto fue observado mediante dos tipos de preguntas-respuestas: el primero, sobre el tiempo global de recorrido, fue espontáneo y sintético, y el segundo, sobre la secuencia del viaje, fue más reflexionado, analítico.

La respuesta al tiempo global de desplazamiento origen-destino es claramente inferior al tiempo que resulta de sumar los tiempos en los tramos de la secuencia de este mismo desplazamiento. En otras palabras, las personas entrevistadas no están muy conscientes del tiempo que emplean en el acreso al transporte colectivo y del tiempo de espera. Los desplazamientos están esencialmente percibidos como ligados a un modo de transporte motorizado. Así que se obtienen respuestas sorprendentes en las que los tiempos totales del mismo viaje pueden variar hasta en 100 por ciento.

\section{Tiempos de desplazamientos largos}

El esquema secuencial de los desplazamientos origen-destino de las personas entrevistadas en Chalco pone en evidencia tiempos muy largos: 10 casos superiores o iguales a una y media horas pudiendo alcanzar tres horas; cuatro casos entre 75 minutos y hora y media, y seis casos inferiores a $\mathbf{3 0}$ minutos. La duración de cada viaje repartida según el tipo de desplazamiento realizado, su duración y su secuencia, se pueden observar en la gráfica 1.

Los viajes más largos (superiores a una hora y media) son los más numerosos. Estos últimos atraviesan el Distrito Federal para llegar a los municipios de Naucalpan, Atizapán de Zaragoza, etc., o a delegaciones como Gustavo A. Madero, Tlalpan e Iztapalapa. Los viajes intermedios (entre 30 y 90 minutos) están asociados a trayectorias que se dirigen esencialmente hasta el centro de la ciudad de México y, excepcionalmente, a las zonas vezinas como Iztapalapa. En cambio, los casos inferiores a 30 minutos corresponden a los traslados circunscritos al municipio de Chalco, es decir, hasta el centro o al interior de la misma colonia (frecuentemente para las compras) y, en menor medida, hasta las delegaciones o los municipios limítrofes como Iztapalapa, La Paz y Tlalmanalco. 
GRÁFICA 1

Tiempos de desplazamiento

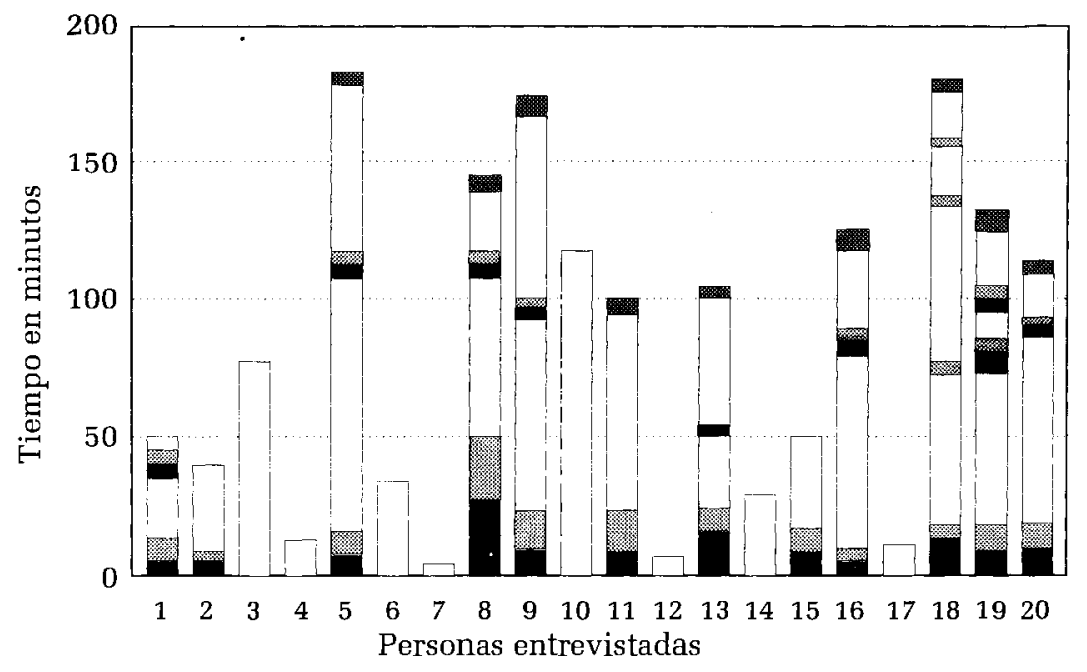

- Hasta la parada

De espera

Desplazamiento

Hasta el destino

Si es cierto que el tiempo de viaje aumenta con la distancia, también se puede observar un aumento del tiempo conforme aumenta la cantidad de modos de transporte usados: el diseño de las rutas también afecta los desplazamientos. Así, la Delegación Iztapalapa aparece en los tres rangos de tiempo. En otra evidencia, dos casos tienen por destino la Central de Abasto: uno de los albañiles toma sólo el suburbano en el centro de Chalco, lo que explica que su tiempo de desplazamiento sea de hora y media; en cambio, un carretillero invierte dos horas de viaje ya que usa dos modos de transporte para ir a la Central de Abasto. En otras palabras, la mala estructuración de las rutas de transporte (y más en lo general, la falta de integración del sistema de transporte) obliga a realizar transbordos entre varios modos de transporte y ello incrementa el tiempo de traslado.

Cabe insistir en que los largos traslados significan para esta población un enorme costo en tiempo y dinero, además de un gran cansancio. Los largos viajes se explican en parte por el tamaño de 
a Zona Metropolitana. Pero el tiempo excesivo también se debe al nal estado de la vialidad y a la baja frecuencia de paso (particularnente de los autobuses) que caracteriza a la periferia. Fatigosos raslados, mediante un transporte saturado y lento, equivalen a jue la jornada de trabajo aumente de manera considerable. Adenás, las deficiencias en la baja frecuencia de paso, capacidad relucida y falta de cobertura, obligan a los trabajadores a salir más emprano de sus casas (y dormir menos) para llegar al primer molo de transporte que está a su alcance. Ello se debe a que requieren iempo de holgura para afrontar eventuales retrasos o imposibililad de tomar un transporte, lo que se traduciría en llegar tarde a ;us trabajos.

Tiempo de acceso al transporte colectivo

玉l análisis de los tiempos de acceso a los diferentes modos de ransporte colectivo en Chalco conlleva informaciones complenentarias a la oferta de transporte existente. En efecto, este análiis permite distinguir dentro de Chalco las zonas peor cubiertas que se traducen en un tiempo de acceso al transporte comprendido entre 15 y 30 minutos, o 1 a 3 kilómetros), y en contraste, zoaas con buena cobertura (con tiempos de acceso inferiores a diez minutos). Con base en las entrevistas ya mencionadas se elaboró эl cuadro 8 que muestra los tiempos de recorrido a pie al primer modo de transporte opcional.

En el cuadro 8 se puede observar que los tiempos de acceso más largos al transporte colectivo (o sea con un deficiente acceso a este transporte) concierne a los habitantes cuyo lugar de residencia es lejano de la autopista México-Puebla, como en el caso que se da en la colonia San Miguel Xico (entrevista núm. 17) que, para llegar a algún modo de transporte público, requieren caminar veinte minutos. Similarmente, tienen dificultades los residentes de las zonas recientemente urbanizadas (i.e. con una antigüedad menor o igual a cuatro años), donde el transporte no penetra como en el caso del mayor tiempo de acceso que se da en la colonia Nueva San Isidro (entrevista núm. 8) que sólo tiene acceso al autobús de Ruta 100 o al suburbano, pero mediante un recorrido a pie de 30 minutos.

Al contrario, los tiempos de acceso más cortos se observan en las zonas más antiguamente urbanizadas como el Chalco antiguo o en las zonas ubicadas cerca de una avenida como las avenidas Alfredo del Mazo, Isidro Fabela, Emiliano Zapata y López Mateos. Al interior de una misma colonia se pueden notar diferentes tiempos 
de acceso al transporte. El tiempo de acceso "bueno o malo" dependerá de la proximidad de la vivienda a una avenida por donde circula el transporte. Por ejemplo, esto da lugar a que se tengan respuestas tan diferentes dependiendo de que se viva en la parte norte (entrevistas 11 y 14) o en la parte sur (entrevistas 10 y 13) de la colonia Covadonga. ${ }^{5}$ No obstante, en todos esos casos se camina al menos diez minutos.

El cuadro 8 muestra también tiempos de acceso diferentes en función de los distintos modos de transporte. Así, algunas personas entrevistadas estiman, con razón, que un transporte colectivo demasiado lejano a su vivienda significa un no acceso a este transporte. Éste es el caso de parte de los habitantes de la colonia Covadonga, que no tienen acceso al suburbano, o el caso de la colonia Nueva San Isidro que no tiene fácil acceso a los "micro" y (o) a las "combis". Asimismo, los habitantes de Chalco antiguo no pueden acceder a la Ruta 100. Los tiempos de acceso largos o cortos, o aun el no acceso, van a determinar, en muchos casos, la decisión del primer modo de transporte a usar para salir de la zona, aunque esto está influido también por otros factores (véase el análisis de la sección siguiente).

\section{Desplazamientos y modos de transporte usados}

El uso de un cierto modo de transporte colectivo está determinado por la oferta, el acceso, la tarifa, etc., pero igualmente por la racionalidad que guía la selección del usuario, y esto está en función de su experiencia urbana y su pertenencia a un estrato social.

El cuadro 9 indica la secuencia de modos de transporte usados por los entrevistados. En ese cuadro se muestra una predominancia en cuanto al uso del transporte suburbano como primer modo. El taxi colectivo ("micros" y "combis") y el autobús Ruta 100 aparecieron con menor frecuencia en relación con el suburbano. El Metro predomina como segundo modo de transporte más usado. Ello se explica por dos razones esenciales: lo económico (40 centavos) y por el diseño de las rutas de los transportes concesionados que, básicamente, alimentan las estaciones del Metro.

Se observa también que poco más de la mitad de los traslados se realiza mediante un solo modo de desplazamiento. De éstos,

5 Como ya se describió, varias rutas del transporte colectivo circulan en la avenida ubicada al norte de la colonia Covadonga y al sur de Tres Marías, enlazando la avenida López Mateos y el centro de Chalco (la llamada Vía de Prestigio), pero no penetran en las colonias adyacentes. 
EUADRO 8

Tiempos de acceso a los diferentes modos de transporte colectivo

\begin{tabular}{cccc}
\hline Entrevista & & & $\begin{array}{c}\text { Taxis } \\
\text { colectivos: } \\
\text { microbuses } \\
\text { y combis }\end{array}$ \\
\hline 1 & Autobús $R-100$ & $\begin{array}{c}\text { Autobús } \\
\text { suburbano }\end{array}$ & $20 \mathrm{~min}$ \\
2 & $25 \mathrm{~min}$ & $10 \mathrm{~min}$ & $5 \mathrm{~min}$ \\
3 & $5 \mathrm{~min}$ & $/$ & $15 \mathrm{~min}$ \\
4 & $15 \mathrm{~min}$ & $/$ & $5 \mathrm{~min}$ \\
5 & $5 \mathrm{~min}$ & $/$ & $10 \mathrm{~min}$ \\
6 & $10 \mathrm{~min}$ & $/$ & $20 \mathrm{~min}$ \\
7 & $10 \mathrm{~min}$ & $/$ & $10 \mathrm{~min}$ \\
8 & $20 \mathrm{~min}$ & $30 \mathrm{~min}$ & $15 \mathrm{~min}$ \\
9 & $30 \mathrm{~min}$ & $5 \mathrm{~min}$ & $10 \mathrm{~min}$ \\
10 & $20 \mathrm{~min}$ & $10 \mathrm{~min}$ & $10 \mathrm{~min}$ \\
11 & $20 \mathrm{~min}$ & $10 \mathrm{~min}$ & $/$ \\
12 & $10 \mathrm{~min}$ & $5 \mathrm{~min}$ & $10 \mathrm{~min}$ \\
13 & $/$ & $20 \mathrm{~min}$ & $10 \mathrm{~min}$ \\
14 & $/$ & $10 \mathrm{~min}$ & $10 \mathrm{~min}$ \\
15 & $/$ & $10 \mathrm{~min}$ & $20 \mathrm{~min}$ \\
16 & $10 \mathrm{~min}$ & $5 \mathrm{~min}$ & $10 \mathrm{~min}$ \\
17 & $20 \mathrm{~min}$ & $20 \mathrm{~min}$ & $15 \mathrm{~min}$ \\
18 & $20 \mathrm{~min}$ & $10 \mathrm{~min}$ & $10 \mathrm{~min}$ \\
19 & $15 \mathrm{~min}$ & $15 \mathrm{~min}$ & \\
20 & $15 \mathrm{~min}$ & $10 \mathrm{~min}$ & \\
\hline
\end{tabular}

1 Véase cuadro 7.

Nota: la diagonal significa que no está a su alcance.

seis representan viajes en transporte no motorizado, a pie o en bicicleta, y se limitan al municipio de Chalco, y cinco más se hacen en transporte motorizado: en automóvil y en autobús suburbano. Estos últimos pueden salir de los límites del municipio. Lógicamente, el uso de más de un medio de transporte corresponde a desplazamientos de larga distancia.

En particular, el primer criterio que parece guiar la selección del modo de transporte es el tiempo de acceso al mismo. En el cuadro 9 se encuentra que sólo tres de veinte casos (entrevistas 1, 18 y 20) no tienen una explicación evidente del porqué no escogen un modo de transporte público que les implique minimizar el tiempo de recorrido a pie para abordarlo. Quizás se deba a que abordan un segundo modo de transporte y así sea el tiempo total del viaje lo que más les conviene de esa cadena de transporte, o puede ser también la baja tarifa de Ruta 100 (en dos casos) o algún otro factor de tipo cualitativo (véase el análisis posterior). En cambio, en el 
resto de los casos hay una explicación de la racionalidad de la decisión. En primer lugar, hay seis casos en los que los usuarios realizan su viaje a pie o en bicicleta, y ello se debe a que son viajes de corto recorrido (máximo 30 minutos). Otros tres casos optan por medios de transporte motorizados pero propios ( $\operatorname{casos} 2,3 \mathrm{y}$ 10). Así, queda un total de ocho viajes fuera de la región, en los que los habitantes de Chalco seleccionan el modo de transporte público y motorizado que les representa el menor tiempo de acceso a pie. En otras palabras, sólo en tres de doce veces se observa que este factor no es determinante del modo de transporte y no hay una explicación evidente.

Para ilustrar de una manera más clara lo anterior se detallarán algunos de los casos. Así, para ejemplificar la elección de un modo de transporte determinada por su proximidad, se tiene el caso del estudiante que reside en la colonia Culturas de México y que, para ir a la UNAM, usa el suburbano (y después el Metro) porque "es lo más cerca de mi casa" con un tiempo de acceso de 5 minutos, en tanto que la Ruta 100 demora 20 minutos y el "pesero" 15.

Sin embargo, también se pueden observar casos en los que el acceso al modo de transporte no es crucial. El vendedor de Santa Cruz se traslada en microbús hasta Tlalmanalco aunque el tiempo de acceso a éste es 20 minutos: para él, el taxi colectivo es "más rápido". Así, lo que el usuario valoriza es la velocidad del medio de transporte en este recorrido y no el acceso al mismo. El soldador de la colonia Guadalupana se dirige a Iztapalapa en Ruta 100 y en Metro porque "es más económico", aunque tenga que caminar más. Igualmente, una "elección" que se puede considerar $a$ priori como irracional, en realidad no lo es. Este es el caso del ama de casa de la colonia Nueva San Isidro, quien para hacer sus compras se traslada hasta el centro de la ciudad de México (La Merced). Ella usa el suburbano y no la Ruta 100 (ambos ubicados a más de dos kilómetros de su casa), con una tarifa dos veces más elevada que la Ruta 100. Lo extraño es que si bien la frecuencia de paso de la Ruta 100 es claramente inferior (en ocasiones más de media hora) a la del suburbano, la Ruta 100 realiza su recorrido más rapidamente. Lo que podemos suponer es que esta ama de casa va a valorizar su tiempo de espera y entonces va a usar el primer modo de transporte que pase, y en este caso es el suburbano.

La imposibilidad de elección se expresa claramente en el ejemplo del machetero de la colonia Covadonga, que se desplaza hasta la Delegación Miguel Hidalgo en suburbano (y en Metro), porque es el único medio de transporte al cual tiene acceso, y a más de un kilómetro de su vivienda. 
Por otra parte, es importante analizar los desplazamientos no notorizados. Si bien es lógico que los desplazamientos con destito en las proximidades del domicilio (menos de un kilómetro) se ealicen a pie, resulta interesante observar desplazamientos más argos, de dos a tres kilómetros. Tal es el caso del ama de casa que va a pie al centro de Chalco (30 minutos) para hacer sus compras y regresa en microbús o en taxi si le "queda más dinero".

Esos desplazamientos a pie son particularmente importantes $\mathrm{m}$ las zonas recientemente urbanizadas, donde la oferta de transjorte es muy deficiente.

Dos terceras partes de las personas entrevistadas afirman que su familia posee de una a tres bicicletas. Los viajes en bicicleta se sircunscriben al municipio de Chalco. Es el caso del zapatero de la colonia Culturas de México que va en diez minutos (aproximadamente dos kilómetros) al centro de Chalco para su trabajo; o эun el albañil de la colonia Covadonga que va a trabajar a $30 \mathrm{mi}-$ autos (tres a cuatro kilómetros) a la colonia Nueva San Isidro. Estos desplazamientos a pie o en bicicleta no son facilitados por la infraestructura: banquetas frecuentemente inexistentes, calles no pavimentadas, lodosas o polvosas (ya no se piense en carriles exclusivos o señalización adecuada), falta de iluminación nocturna, etcétera.

\section{Comparación ida y regreso}

Los desplazamientos de ida y regreso frecuentemente son los mismos y responden a la misma lógica que de ida. La razón más invocada está ligada a la proximidad del transporte: "es más cerca de mi trabajo", "me deja cerca de mi casa"; o aun "es más rápido". Así, se observaron cadenas de desplazamientos como Ruta 100Metro-suburbano, de ida, que se invierten para el regreso, suburbano-Metro-Ruta 100.

En ocasiones el regreso puede ser diferente. Esto se observa de manera muy frecuente cuando se trata de un motivo de compras ("al mercado"): ida a pie y regreso en transporte motorizado (por el peso del "mandado"). Sin embargo, si el motivo del viaje es el trabajo, el cambio del modo empleado para el regreso es más raro. Éste es el caso del obrero de la colonia Santiago, que va a usar el suburbano, el Metro (con un transbordo), y la Ruta 100, para llegar a su lugar de trabajo en Naucalpan (tres horas). Su regreso es diferente: el Metro hasta Pantitlán, después el tren ligero hasta Santa Martha, y el microbús. Su regreso es más largo y la razón por la cual él modifica su cadena de desplazamiento es la 
CUADRO 9

Modos de desplazamiento usados

\begin{tabular}{|c|c|c|c|c|}
\hline Caso $^{1}$ & $\begin{array}{l}\text { Primer } \\
\text { modo }\end{array}$ & Segundo & Tercero & Cuarto \\
\hline 1 & Pesero & Pesero & 1 & 1 \\
\hline 2 & Camión de empresa & 1 & 1 & 1 \\
\hline 3 & Auto particular & 1 & 1 & 1 \\
\hline 4 & A pie & 1 & 1 & 1 \\
\hline 5 & Ruta 100 & Metro & 1 & 1 \\
\hline 6 & A pie & 1 & 1 & 1 \\
\hline 7 & A pie & 1 & 1 & 1 \\
\hline 8 & Suburbano & Metro & 1 & 1 \\
\hline 9 & Suburbano & Metro & 1 & 1 \\
\hline 10 & Auto particular & 1 & 1 & 1 \\
\hline 11 & Suburbano & I & I & 1 \\
\hline 12 & Bicicleta & 1 & I & 1 \\
\hline 13 & Suburbano & Metro & 1 & 1 \\
\hline 14 & Bicicleta & 1 & 1 & 1 \\
\hline 15 & Suburbano & 1 & I & 1 \\
\hline 16 & Suburbano & Pesero & I & I \\
\hline 17 & A pie & 1 & 1 & 1 \\
\hline 18 & Ruta 100 & Metro & Metro & Suburbano \\
\hline 19 & Suburbano & Metro & Metro & 1 \\
\hline 20 & Ruta 100 & Metro & 1 & 1 \\
\hline
\end{tabular}

1 Véase cuadro 7.

Nota: la diagonal significa que no está a su alcance.

congestión ("el amontonamiento") en el autobús suburbano (a las 8:00 horas p.m.). Así, elimina este medio y usa la combinación tren ligero-microbús.

\section{Las alternativas de desplazamiento}

Desafortunadamente, en la actualidad hay muy pocas alternativas para los habitantes de Chalco, según sus propias palabras. Para los raros propietarios de un automóvil, la alternativa obligada corresponde al día "hoy no circula" o cuando el coche está descompuesto. Para el estudiante de la UNAM, su alternativa es el transporte universitario, pero lo usa poco porque "tarda mucho". Esto representa una alternativa económica que él va a usar cuando no cuenta con suficiente dinero.

En la medida en que penetramos en el Distrito Federal, el número de alternativas posibles aumenta porque la oferta es más 
mplia y más diversificada, lo que no es el caso del Estado de Méico y menos aún en el valle de Chalco.

Por otra parte, sabiendo que las tarifas del transporte colectio en el Estado de México son dos o tres veces más altas que en el Jistrito Federal, la parte del presupuesto destinada al transporte to es despreciable.

El chofer de camión de carga usa la combinación Metro-Ruta 00 (1.60 nuevos pesos al día, ida y regreso), lo que representa aproimadamente 40 nuevos pesos al mes, o sea, menos de $10 \%$ del salaio mínimo. Él usa transporte subvencionado, por lo que sus gastos $\mathrm{n}$ transporte no representan una parte muy importante de su presuuesto. No es el caso del obrero que se traslada en suburbano-metro le ida (2.40 nuevos pesos) y Metro-tren ligero-pesero (2.70 nuevos ıesos), o sea más de 120 nuevos pesos al mes, más de $25 \%$ del salaio mínimo.

En Chalco, los peseros y suburbanos son considerados como nodos de transporte "caros". Un transporte "caro" y de "mala caidad" puede explicar, en parte, el escaso número de desplazaaientos ligados a la diversión. En efecto, de las veinte personas ntrevistadas, quince afirman no tener ninguna diversión; otros asos, los que tienen alguna, juegan futbol o realizan visitas y se lesplazan a pie, frecuentemente.

\section{le la relación transporte y desarrollo urbano}

Iquí, cabe recuperar la hipótesis planteada en la introducción: la expansión de la ciudad de México hacia la zona de Chalco se lebe a las facilidades que otorga un buen sistema de transporte? in verdad, la respuesta no parece ser lineal y merece ser tratada on más profundidad. Nuestro análisis preliminar indica que, en ealidad, la cercanía de Chalco con la autopista México-Puebla rarece ser un factor determinante para los primeros asentarmienos. Sin embargo, en la actualidad, para la gran mayoría de los haitantes no existe una accesibilidad realmente atractiva, salvo paa un sector reducido de ellos. Entonces, los asentamientos más ien parecen deberse al bajo costo de los terrenos. Para mediados le 1993 era factible comprar un terreno baldío de 200 metros (sin Irenaje, agua potable suficiente, banquetas, pavimentación, etc.) $\mathrm{n}$ alrededor de 15000 nuevos pesos.

Entonces, tampoco parece cumplirse la proposición teórica de .owdon Wingo en el sentido de que la localización urbana residenial representa el punto de equilibrio entre costos de transporte y ostos del suelo periférico. La razón estriba en que los usuarios 
están pagando altos costos de transporte (no sólo monetarios sino en tiempo, incomodidad y riesgos), que se acumulan y pueden alcanzar montos muy altos cuando se considera el largo plazo. Así, su decisión no corresponde a una lógica o racionalidad minimizadora de costos a largo plazo. En realidad, se asumen los altos costos de transporte porque no hay alternativa: el precio de los terrenos, departamentos o casas en las áreas urbanizadas de la ciudad están fuera de su alcance.

\section{Conclusiones}

Las principales conclusiones de este trabajo se pueden agrupar en dos aspectos: calidad del servicio de transporte y política de transporte.

\section{De la infraestructura y el servicio de transporte}

Si se define la calidad de la cobertura del transporte colectivo con base en la accesibilidad espacial, al tiempo, a la comodidad y a la velocidad comercial con que se efectúan los viajes, se puede afirmar que el servicio de transporte en el municipio de Chalco es un servicio definitivamente malo. En efecto, el transporte en esta zona presenta graves problemas. Las dificultades de acceso de los vehículos de transporte colectivo se deben no sólo a la escasez de arterias sino también al mal estado de las vías de circulación. En este trabajo se encontró que esta falta de accesibilidad obliga a los habitantes de Chalco que van al Distrito Federal a trabajar (lo que es el caso de más de $60 \%$ de la población), y que no tienen la suerte de vivir próximos a una carretera donde circula un transporte colectivo, a efectuar largos desplazamientos a pie (en muchos casos de media hora).

Aunque Chalco tiene acceso básicamente a tres vías importantes hacia el Distrito Federal (autopista México-Puebla, carretera México-Puebla y carretera México-Tláhuac), estas vías tienen otras prioridades de comunicación interurbana. Ademas, la zona presenta una cobertura insuficiente en transporte colectivo. Así, es posible observar los embotellamientos y el congestionamiento de los usuarios del transporte colectivo a las horas de máxima demanda y más particularmente en la mañana. Por consiguiente, los tiempos de viaje pueden llegar hasta tres horas (solamente en el viaje de salida). Además de esas condiciones de desplazamiento deficientes, el comportamiento a veces agresivo y abusivo (cobro arbitrario de tarifas) de los choferes, hacen de los desplazamientos 
$m$ verdadero desafío para quien quiere o debe ir a la ciudad para iu trabajo, sus compras o sus estudios.

De hecho, la comparación de los atributos del servicio que se Jfrece en Chalco muestra que se está muy por debajo de las ya de jor sí deficientes condiciones del transporte que en general se ob¡erva en el resto del Área Metropolitana de la Ciudad de México. Así, los tiempos de espera, de caminata para abordar el transpore, los costos monetarios y los tiempos de desplazamiento son narcadamente mayores para los habitantes de Chalco, lo que se maaifiesta en una opinión también mucho más adversa que la observada por el resto de usuarios de la ciudad.

Un hecho importante que encuentra este estudio consiste en que, para los habitantes de Chalco, más que el valor del tiempo que se consume en los desplazamientos y la incomodidad que representa viajar en transporte colectivo, el problema esencial es la posibilidad misma de tener acceso al transporte. La noción de accesibilidad toma aquí toda su fuerza porque está lejos de estar asegurada para todos los habitantes.

La dependencia de los desplazamientos a pie, se debe, por un lado, a la insuficiencia de las redes de transporte colectivo que no cubren, o cubren mal los barrios periféricos, tienen horarios irregulares y generalmente saturados. Por otro lado, se debe también a los pocos recursos de los usuarios en esta zona, que impiden o limitan un uso más frecuente de los transportes colectivos -los motivos de viaje se limitan al trabajo, las compras y el estudio-; y muy escasamente se presentan desplazamientos ligados a las diversiones.

\section{De la política de transporte}

En esencia, de lo anterior se manifiesta un problema de equidad social, y el concepto de "derecho al transporte" adquiere aquí todo su valor. En particular, el transporte debe concebirse en sí mismo como parte de la calidad de vida, pero también juega un papel muy importante para tener acceso a diversas oportunidades de incrementar en forma real el nivel de vida de las personas. Así, el objetivo principal de toda política de transporte urbano debiera ser el de asegurar a todos los habitantes de la ciudad las mismas posibilidades de acceder a las diferentes zonas de la ciudad. Esta accesibilidad mínima supone una red de vialidad que permita al transporte colectivo cubrir el espacio urbano y, también, el establecimiento de medidas que faciliten los desplazamientos no motorizados -a pie y bicicleta. Además, supone la existencia de modos de transportes 
adaptados a las posibilidades económicas, considerando bajas inversiones y tarifas moderadas.

Las soluciones a los problemas descritos implican acciones al corto plazo que no son fáciles de realizar (en especial por la falta de recursos de este municipio y de sus recién llegados), pero no pueden postergarse. Entre tales acciones destaca la necesidad de restructurar las rutas de transporte y, asociado con ello, mejorar la vialidad por las arterias prioritarias. En particular, la extensión de las rutas de la Ruta 100 parecen lo más deseado por los usuarios, pero ello deberá estar acompañado de un aumento de la cantidad de autobuses asignados a tales rutas. De ser así, debe realizarse un cuidadoso tratamiento "político" del problema porque pueden afectarse intereses económicos de los transportistas suburbanos y "peseros". Para evitar un conflicto, habría que buscar un rediseño de las rutas que también les permita competir con Ruta 100 y dejar que sean los usuarios los que decidan su preferencia.

El problema de ofrecer un transporte barato y subsidiado no puede ser analizado en el espacio de este artículo. Es claro, sin embargo, que se requiere un tratamiento especial y no seguir dejando a las "fuerzas del mercado" la solución del problema de transporte de los habitantes de este suburbio pues las pruebas de su desempeño son claras: se tiene un transporte caro, malo y peligroso. Es difícil argumentar que esto sea un estado de eficiencia. Así, la planeación e intervención estatal parece justificarse ante este inocultable fracaso de las fuerzas del mercado. En ese sentido, destacan las deficiencias infraestructurales que no son atendidas porque no resultarían un negocio realmente atractivo. Por ejemplo, es impostergable, en particular, la construcción del camino Chalco-Tláhuac cuya condición actual ha causado ya la pérdida de vidas humanas sin que se haya logrado sensibilizar a las correspondientes autoridades.

Además, es claro que buena parte de los problemas se deben al crecimiento explosivo y anárquico de la zona. En este sentido, se requiere también de una visión de mediano y largo plazos, pues sólo con la aplicación estricta de planes de desarrollo urbano más realistas podría esperarse un crecimiento más ordenado. Es ya innegable que este municipio va a seguir creciendo en las zonas hoy todavía disponibles. Podría ser tiempo para prever condiciones de transporte -y por tanto, de vida- más justas y dignas para los también mexicanos que habitan este municipio. 


\section{Bibliografía}

Altshuler, Alan (1979), Current Issues in Transportation Policy, Lexington (Mass.), Lexington Books.

Anderson, Neis (1974), Sociología de la comunidad urbana, México, Fondo de Cultura Económica.

Departamento del Distrito Federal (1993), Anuario de transporte y vialidad, 1991, Coordinación General del Transporte.

Dickey, John W. (ed.) (1978), Manual del transporte urbano, Madrid, Instituto de Estudios de Administración Local.

García Mora, Carlos (1981), Naturaleza y sociedad en Chalco-Amecameca, México, Biblioteca Enciclopédica del Estado de México, 97.

Instituto Nacional de Estadística, Geografía e Informática (1990), XI Censo General de Población y Vivienda, 1990, datos por AGEB urbano, Estado de México.

Islas, Víctor M. (1983), Diseño de rutas de transporte, tesis de Ingeniería de Transporte, México, UPIICSA-IPN.

- (1990), "Manual de estudios de transporte urbano", México, Secretaría de Educación Pública (mimeo).

- (1991), Efectos de la subvención, la regulación y las formas de propiedad del transporte colectivo urbano sobre su eficiencia y calidad: el caso de la ciudad de México, informe preparado para la CEPAL en febrero de 1991 (núm. LC.IN.115).

- (1993), "Diagnóstico general del transporte urbano en la ciudad de México", Serie Documentos de Investigación del Procientec DI-8, México, El Colegio de México, noviembre.

Juárez, J.M. y R. Martínez (1990), Encuesta Socioeconómica, agosto,.oRsTOM/UAM/CEE.

Krueckeberg y Silvers (1978), Análisis de Planificación Urbana, México, Limusa.

Lane, R., T. Powell y P. Smith (1978), Planificación analítica del transporte, Madrid, Instituto de Estudios de Administración Local (Nuevo Urbanismo, 1).

Lindón Villoría, Alicia (1991), "La periferia metropolitana y la informalidad: el caso de Chalco", tesis de maestría en Desarrollo Urbano, México, El Colegio de México.

Meyer, John R. y José A. Gómez I. (1981), Autos, Transit and Cities, Cambridge (Mass.), Harvard University Press.

Mitchell, Robert B. y Chester Rapkin (1954), Urban Traffic. A function of: Land Use, Nueva York, Columbia University Press.

Owen, Wilfred (1972), The Accesible City, Washington D.C., The Brookings Institution.

Proyecto Chalco (1990), reportes diversos, México, ORSTOM/UAM/CEE. 
Rosales Montana (1988), De la mobilité-flux a la mobilité-enjeux, tesis de doctorado en Ciencias Económicas, Lyon.

Roschlau, Michael W. (1981), Urban Transport in Developing Countries: The Peseros of México City, Vancouver (Canadá), Centre for Transportation Studies.

Schteingart, Martha (coord.) (1991), Espacio y vivienda en la ciudad de México, México, El Colegio de México.

Valero Calvete, Javier (1976), Transportes urbanos, Madrid, Dossat.

Wingo, Lowdon (1972), Transporte y suelo urbano, Barcelona, Oikos-Tau. 